\title{
Quantitative Evaluation of the Lifshitz-Type Temperature Effect on the Casimir Force
}

\author{
Frédéric Schuller ${ }^{1}$, Renaud Savalle ${ }^{2}$ \\ ${ }^{1}$ Laboratoire de Physique des Lasers, Villetaneuse, France \\ ${ }^{2} \mathrm{CNRS} /$ Observatoire de Paris-Meudon, Paris, France \\ Email: renaud.savalle@obspm.fr
}

How to cite this paper: Schuller, F. and Savalle, R. (2017) Quantitative Evaluation of the Lifshitz-Type Temperature Effect on the Casimir Force. Journal of Modern Physics, 8, 1779-1783.

https://doi.org/10.4236/jmp.2017.811105

Received: September 12, 2017

Accepted: October 8, 2017

Published: October 11, 2017

Copyright (c) 2017 by authors and Scientific Research Publishing Inc. This work is licensed under the Creative Commons Attribution International License (CC BY 4.0).

http://creativecommons.org/licenses/by/4.0/ (c) (i) Open Access

\begin{abstract}
We consider the extension of the Casimir effect to finite temperatures in the ideal case of perfectly reflecting plates. We apply Lifshitz's theory in its Dzyaloshinskii version, and calculate the resulting force numerically for various plate distances. We show that the limiting expression found in the literature corresponds to unrealistic values of the parameters for which the force is too small to be measurable. Preliminary remark: There exists a huge literature on the Casimir effect both theoretical and experimental. In this note we concentrate on a particular point of the subject, quoting only references directly related to this point.
\end{abstract}

\section{Keywords}

Quantum Vacuum, Quantum Statistics, Thermal Radiation, Zero-Point Energy, van der Waals Forces

\section{Introduction}

We consider the ideal situation of two parallel metallic plates presenting in the gap between them a reflection power of $100 \%$ and containing a vacuum at absolute zero temperature. In the spirit of Casimir's initial work [1] the attractive force between the plates should give macroscopic access to the zero-point energy in the vacuum. Naturally these conditions are never met in nature, but as a minimum extension, the zero temperature can be removed. As is well-known, the Lifshitz theory of the force between dielectric bodies yields, with dielectric constants taken to infinity, in the limit $T \rightarrow 0$ Casimir's result

$$
F_{0}=\frac{\pi^{2}}{240} \frac{\hbar c}{a^{4}}
$$


with $a$ the plate distance, and the force that per unit area between the plates. In the case of finite temperatures we apply here a version of Lifshitz's theory due to Dzyaloshinskii and Pitaevskii (1959) and discuss numerical results not found so far in the literature.

\section{The Lifshitz Calculation}

As reported in ref [2], the Lifshitz force between parallel dielectric plates is derived by introducing Matsubara Green's functions involving the Matsubara pseudo-frequency

$$
\varsigma_{s}=2 \pi s k_{B} T / \hbar
$$

Considering the limiting case of perfect metals, with dielectric constants taken to infinity and $F_{0}$ the Casimir value of Equation (1), this expression leads to the result

$$
\frac{F(a)}{F_{0}(a)}=R=\frac{60}{\pi^{3}} \kappa a T \sum_{s=0}^{\infty} \int_{1}^{\infty} \frac{(2 \pi s \kappa a T p)^{3} \mathrm{~d} p}{p[\exp (4 \pi s \kappa a T p)-1]}
$$

with the value of the parameter $\kappa=\frac{k_{B}}{\hbar c}=4.36 \times 10^{2} \mathrm{~K}^{-1} \cdot \mathrm{m}^{-1}$, and the prime indicating that for the term with $s=0$ a factor $1 / 2$ has to be inserted.

A derivation of the Equation (3) can be found in many places not listed here.

Notice that this expression depends on the combined parameter $a T$.

Making the substitution $\delta s=1=(\hbar / 2 \pi T) \mathrm{d} \varsigma$, valid for $\rightarrow 0$, the resulting double integral over $p$ and $\zeta$ can be done analytically, yielding unity for the $R$ value.

As a first step we have evaluated the quantity $R$ numerically in ref [3] without the term $s=0$. The results, designated as $R_{A}=\frac{F_{A}}{F_{0}}$ are shown on Figure 1(a), representing the variation of this quantity with temperature for several plate distances.

The term $s=0$ has to be calculated separately. Taking the proper limit of the Equation (3) one finds as shown in the appendix

$$
R_{B}=\frac{F_{B}}{F_{0}}=\frac{30}{\pi^{3}} \kappa a T \times 2.404=2.326 \kappa a T=1014 a T
$$

Adding these values to the results of Figure 1(a), we obtain for $R=R_{A}+R_{B}$ the curves of Figure 1(b) showing an increase of the force with temperature. In the case, with the highest value i.e. $a T=5 \times 10^{-3}$, the Equation (4) applies, since in this case $R_{A}$ is negligibly small. This limit, corresponding to a linear temperature variation of the force, can be found in the literature [4] [5]. It is however nonrealistic since much smaller distances must be used to obtain a measurable force.

\section{Discussion}

In actual experiments one is far away from the ideal conditions presupposed in these calculations. In particular, in order to obtain small separation distances, 


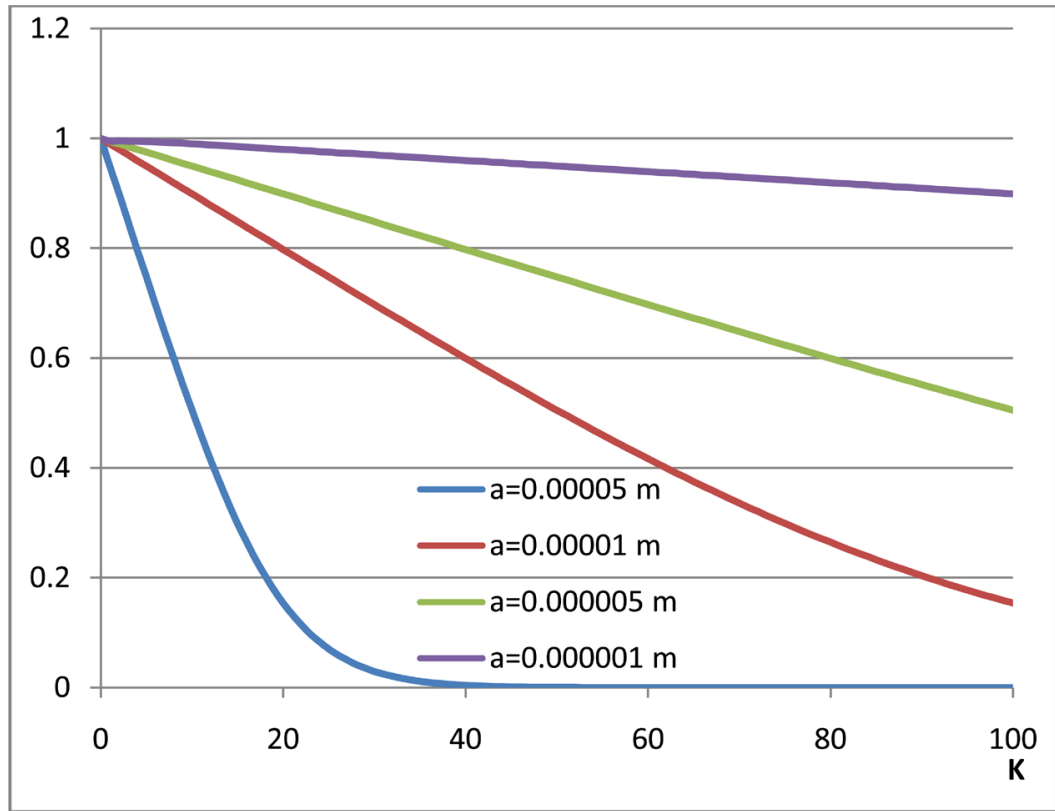

(a)

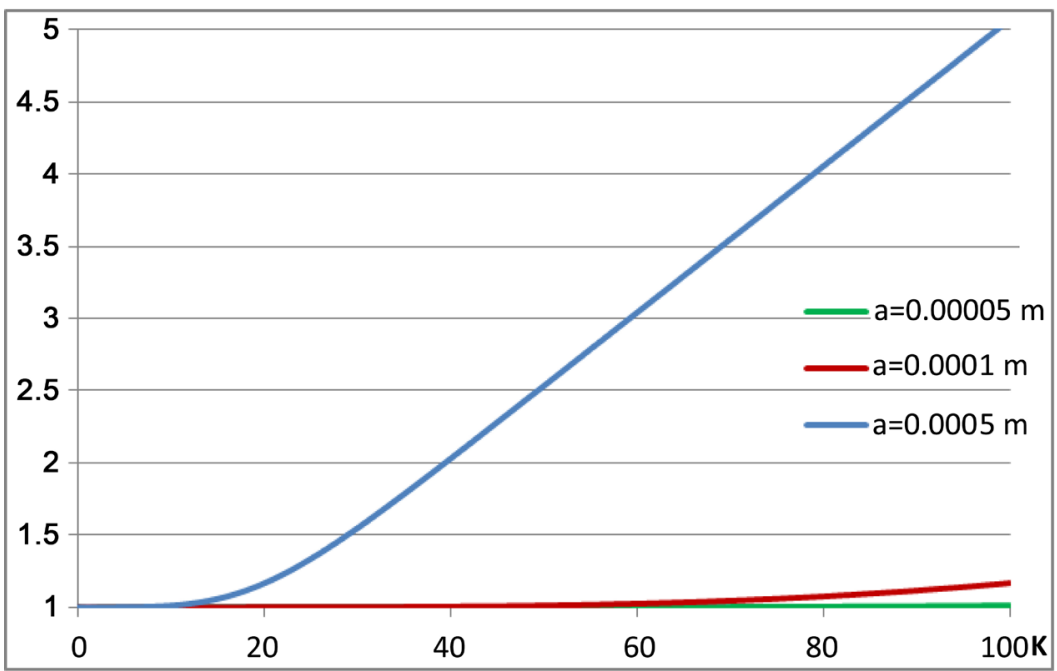

(b)

Figure 1. (a) Ratio $R_{A}=\frac{F_{A}(a)}{F_{0}(a)}$ as function of temperature for several values of plate separations; (b) Ratio $R=R_{A}+R_{R}$ for the same parameters as in Figure 1(a).

very special geometries have to be used. Despite these facts it seems that the temperature effect is still at the limit of observability, although progress has been made as shown e.g. in [6].

According to our results, as long as very small distances are involved, temperatures up to room temperature don't compromise the observation of the force. This could be different if measurements at larger distances could be made. In that case a force-increasing temperature effect would be more visible. Note that during the last 10 years, mostly experimental results have been presented for which a complete list can be found in reference [6]. 


\section{Conclusions}

In this note we only want to stress the fact that a strict application of Lifshitz's theory in its Dzyaloshinskii version confirms the older results obtained by more conventional methods.

But in addition we present exact numerical results, not found so far in the literature. However, the usual discussion of the temperature effect does not show clearly the fact that limiting expressions do not correspond to realistic measurable values of the force.

\section{References}

[1] Casimir, H.G.B. (1948) Proceedings of the Koninklijke Nederlandse Akademie Van Wetenschappen, 57, 61 .

[2] Landau, L.D. and Lifshitz, E.M. (2006) Course of Theoretical Physics. In: Lifshitz, E.M. and Pitaevski, L.P., Eds., Statistical Physics Part 2, Vol. 9, Oxford.

[3] Schuller, F., Savalle, R. and Neumann-Spallart, M. arXiv:1205.6364.

[4] Milton, K.A. (2001) The Casimir Effect. World Scientific, Singapore.

[5] Milonni, P. (1997) The Quantum Vacuum. Academic Press, San Diego, New York, Boston, London, Sydney, Tokyo, Toronto.

[6] Boström, M. and Semelius, B.E. (2000) Physical Review Letters, 84, 20. 


\section{Appendix}

We want the limit of the expression

$$
F(s)=s^{3} \int_{1}^{\infty} \frac{p^{2} \mathrm{~d} p}{\mathrm{e}^{s p}-1}
$$

for $s=0$.

Changing integration variables by setting $s p=u, p=\frac{u}{s}, \mathrm{~d} p=\frac{\mathrm{d} u}{s}$, we have

$$
F(s)=\int_{s}^{\infty} \frac{u^{2} \mathrm{~d} u}{\mathrm{e}^{u}-1}
$$

yielding for $s=0$

$$
F(s=0)=\int_{0}^{\infty} \frac{u^{2} \mathrm{~d} u}{\mathrm{e}^{u}-1}
$$

Using tables of integrals one finds

$$
\int_{0}^{\infty} \frac{u^{2} \mathrm{~d} u}{\mathrm{e}^{u}-1}=\Gamma(3) \zeta(3)
$$

involving Riemann's zeta function. Given the values $\Gamma(3)=2, \zeta(3)=1.202$ one finally obtains

$$
F(s=0)=2 \times 1.202=2.404
$$

This numerical result remains valid if in Equation (1) $s$ is replaced by $x s$ with $x$ any finite positive number. 